\title{
Review of: "Robust statistical evaluation of tumor incidences in long-term rodent carcinogenicity studies: the reformulated poly-k trend test"
}

\author{
Christian Strupp ${ }^{1}$ \\ 1 Gowan Company
}

\begin{abstract}
Potential competing interests: The author declares that he works for an agrochemical company conducting chronic rodent cancer bioassays in the context of human safety assessment. He is further chairing the Human Health Expert Team of CropLife Europe, and agrochemical industry umbrella organization.
\end{abstract}

General comments:

A very good contribution in a time when a combination of regulatory policy and less and less academic education on in vivo studies drives evaluators away from experience-based expert judgment to statistics as a solidly reproducible criterion for decision making. When statistics is used as a screening tool to focus the evaluator on tumor types that need a closer look, pragmatic models (like historically established) may do the job. However when they are becoming the sole basis for judgment on study outcome, their weaknesses need to be analyzed and improvements proposed as done in this article.

I personally would recommend to add a few more sentences for those readers not fully familiar with factors driving survival in carcinogenicity studies. A common error in interpretation is the expectation that toxicity reduces the lifespan and that tumors are the main cause for mortality. In reality, slight toxicity typically leads to reduced body weight development, which in turn significantly prolongs life span of the highest dose group in rodent cancer bioassays. Tumor formation is not the main cause of death, and $>50 \%$ of the animals have at least one tumor at sacrifice towards the end of their natural lifespan.

I particularly welcome the proposal to consider multiple tumors simultaneously, as the mid-term future of toxicology is focused on mechanistic understanding. The co-occurrence of certain tumors is established, and a dependent/combined analysis will likely respond better to the needs of regulators in the near future.

The use of the analysis across species (2.10) may have to be critically evaluated based on tumor type, depending on the protection goal. For example, hepatic enzyme inducing compounds (and those are frequent across chemical classes at the exaggerated doses tested) lead to liver tumors in rats and mice (most sensitive: male mouse), so here this type of analysis would be appropriate. However, the same mode of action frequently leads to secondary thyroid tumors in rats (females most sensitive), but not in mice. Using a combined analysis may in this specific case not be ideal. Similar species differences exist for HPPD-inhibiting compounds and others. So going both ways (i.e. species-by-species and across-species analysis) may be the best approach. 
Minor editorial proposals:

2.1 potential typo?

"tumors can preliminary diagnosed in animals that die or were sacrificed, particularly early" $\rightarrow$ "tumors can only be diagnosed before the scheduled end of the study in animals that die early or are sacrificed due to unacceptable toxicity" ? 